Volume 72 (2016)

Supporting information for article:

EIGER detector: application in macromolecular crystallography

Arnau Casanas, Rangana Warshamanage, Aaron D. Finke, Ezequiel Panepucci, Vincent Olieric, Anne Nöll, Robert Tampé, Stefan Brandstetter, Andreas Förster, Marcus Mueller, Clemens Schulze-Briese, Oliver Bunk and Meitian Wang 


\section{EIGER detector: application in macromolecular crystallography}

Arnau Casanas ${ }^{a}$, Rangana Warshamanage ${ }^{a}$, Aaron D. Finke ${ }^{a}$, Ezequiel Panepuccia, Vincent Olieric ${ }^{a}$, Anne Nöllc ${ }^{c}$, Robert Tampéc, Stefan Brandstetter ${ }^{b}$, Andreas Förster ${ }^{b}$, Marcus Mueller ${ }^{b}$, Clemens Schulze-Briese $^{b}$, Oliver Bunk ${ }^{a}$, Meitian Wang ${ }^{a \star}$

a Swiss Light Source, Paul Scherrer Institute, Villigen, $\mathrm{CH}-5232$, Switzerland

b DECTRIS Ltd., Taefernweg 1, Baden-Dättwil, CH-5405, Switzerland

c Institute of Biochemistry, Biocenter, Goethe University Frankfurt, Max-von-Laue-Str. 9, D60438 Frankfurt am Main, Germany

* Correspondence e-mail: meitian.wang@psi.ch 
Table S1 Data processing statistics (XDS version March 1, 2015) for lys_1 and lys_1 $2 \times 2$ binned data sets (Figure 4).

\begin{tabular}{ccccccc}
\hline $\begin{array}{c}\text { Resolution } \\
(\AA)\end{array}$ & \multicolumn{2}{c}{$R_{\text {meas }}(\%)$} & \multicolumn{2}{c}{$\langle I / \sigma(I)\rangle$} & \multicolumn{2}{c}{$C C_{1 / 2}(\%)$} \\
& $0.05^{\circ}$ & Binned & $0.05^{\circ}$ & Binned & $0.05^{\circ}$ & Binned \\
& $10.05 \mathrm{~s}$ & $2 \times 2$ & $10.05 \mathrm{~s}$ & $2 \times 2$ & $10.05 \mathrm{~s}$ & $2 \times 2$ \\
\hline 4.07 & 3.3 & 3.3 & 63.62 & 64.27 & 99.9 & 99.9 \\
2.93 & 4.2 & 4.2 & 59.92 & 60.17 & 99.9 & 99.9 \\
2.41 & 5.8 & 5.9 & 44.77 & 43.93 & 99.9 & 99.9 \\
2.10 & 7.9 & 8.0 & 33.26 & 32.57 & 99.8 & 99.8 \\
1.88 & 12.2 & 12.6 & 20.93 & 20.51 & 99.7 & 99.6 \\
1.72 & 21.3 & 22.6 & 11.61 & 11.29 & 99.1 & 98.9 \\
1.59 & 34.7 & 38.9 & 7.06 & 6.49 & 97.3 & 96.5 \\
1.49 & 50.7 & 63.2 & 3.61 & 2.95 & 89.0 & 84.5 \\
1.40 & 64.9 & 98.9 & 2.00 & 1.35 & 62.0 & 56.2 \\
Total & 6.7 & 7.2 & 20.96 & 20.46 & 99.9 & 99.9 \\
\hline
\end{tabular}

Table S2 Data processing statistics (XDS version Oct 15, 2015) for lys_1 and lys_2 (SUM40) data sets (Figure 6).

\begin{tabular}{|c|c|c|c|c|c|c|}
\hline \multirow[t]{2}{*}{$\begin{array}{l}\text { Resolution } \\
\text { (A) }\end{array}$} & \multicolumn{2}{|c|}{$R_{\text {meas }}(\%)$} & \multicolumn{2}{|c|}{$\langle I / \sigma(I)\rangle$} & \multicolumn{2}{|c|}{$C C_{1 / 2}(\%)$} \\
\hline & $\begin{array}{l}0.05^{\circ} \\
/ 0.05 \mathrm{~s}\end{array}$ & SUM40 & $\begin{array}{l}0.05^{\circ} \\
10.05 \mathrm{~s}\end{array}$ & SUM40 & $\begin{array}{l}0.05^{\circ} \\
/ 0.05 \mathrm{~s}\end{array}$ & SUM40 \\
\hline 4.07 & 3.1 & 3.0 & 71.05 & 72.17 & 100 & 100 \\
\hline 2.93 & 3.8 & 3.8 & 66.19 & 66.71 & 99.9 & 100 \\
\hline 2.41 & 5.3 & 5.4 & 48.99 & 48.69 & 99.9 & 99.9 \\
\hline 2.10 & 7.2 & 7.4 & 36.41 & 36.03 & 99.9 & 99.8 \\
\hline 1.88 & 10.9 & 11.1 & 23.64 & 23.10 & 99.8 & 99.7 \\
\hline 1.72 & 18.4 & 19.3 & 13.50 & 12.98 & 99.4 & 99.3 \\
\hline 1.59 & 28.4 & 30.4 & 8.70 & 8.22 & 98.3 & 98.0 \\
\hline 1.49 & 42.4 & 45.7 & 4.70 & 4.38 & 92.9 & 91.7 \\
\hline 1.40 & 55.4 & 58.7 & 2.63 & 2.46 & 75.5 & 75.0 \\
\hline Total & 6.3 & 6.3 & 23.28 & 23.04 & 100 & 100 \\
\hline
\end{tabular}


Table S3 Data processing statistics (XDS version Oct 15, 2015) for different summation of frames in lys_2 data set (Figure 7).

\begin{tabular}{|c|c|c|c|c|c|c|}
\hline \multirow{2}{*}{$\begin{array}{c}\text { Resolution } \\
(\AA)\end{array}$} & \multicolumn{6}{|c|}{$R_{\text {meas }}(\%)$} \\
\hline & SUM5 & SUM10 & SUM20 & SUM40 & SUM80 & SUM160 \\
\hline 4.07 & 2.9 & 3.0 & 3.0 & 3.0 & 3.0 & 2.9 \\
\hline 2.93 & 3.6 & 3.8 & 3.8 & 3.8 & 3.8 & 3.7 \\
\hline 2.41 & 5.1 & 5.3 & 5.4 & 5.4 & 5.3 & 5.3 \\
\hline 2.10 & 6.9 & 7.1 & 7.4 & 7.4 & 7.3 & 7.2 \\
\hline 1.88 & 9.9 & 10.0 & 11.1 & 11.1 & 10.9 & 10.9 \\
\hline 1.72 & 16.0 & 15.9 & 18.5 & 19.3 & 19.1 & 19.3 \\
\hline 1.59 & 24.3 & 23.4 & 27 & 30.4 & 30.8 & 31.2 \\
\hline 1.49 & 34.3 & 33.5 & 36.1 & 45.7 & 48.9 & 50.8 \\
\hline 1.40 & 44.8 & 45.3 & 44.6 & 58.7 & 74.3 & 79.6 \\
\hline Total & 5.8 & 5.9 & 6.2 & 6.3 & 6.3 & 6.4 \\
\hline \multirow[t]{2}{*}{$\begin{array}{l}\text { Resolution } \\
(\AA)\end{array}$} & \multicolumn{6}{|c|}{$\langle I / \sigma(I)\rangle$} \\
\hline & SUM5 & SUM10 & SUM20 & SUM40 & SUM80 & SUM160 \\
\hline 4.07 & 75.11 & 72.74 & 71.62 & 72.17 & 72.95 & 73.85 \\
\hline 2.93 & 70.74 & 68.22 & 66.55 & 66.71 & 67.24 & 67.91 \\
\hline 2.41 & 53.19 & 51.64 & 49.09 & 48.69 & 48.95 & 49.21 \\
\hline 2.10 & 40.49 & 39.39 & 36.5 & 36.03 & 36.29 & 36.45 \\
\hline 1.88 & 26.48 & 26.45 & 23.59 & 23.1 & 23.38 & 23.34 \\
\hline 1.72 & 15.20 & 15.42 & 13.75 & 12.98 & 13.08 & 13.02 \\
\hline 1.59 & 9.88 & 10.09 & 9.19 & 8.22 & 8.09 & 8.04 \\
\hline 1.49 & 5.37 & 5.48 & 5.24 & 4.38 & 4.06 & 3.96 \\
\hline 1.40 & 2.92 & 2.86 & 2.92 & 2.46 & 1.98 & 1.89 \\
\hline Total & 25.5 & 24.99 & 23.57 & 23.04 & 23.08 & 23.15 \\
\hline \multirow[t]{2}{*}{$\begin{array}{c}\text { Resolution } \\
(\AA)\end{array}$} & \multicolumn{6}{|c|}{$C C_{1 / 2}(\%)$} \\
\hline & SUM5 & SUM10 & SUM20 & SUM40 & SUM80 & SUM160 \\
\hline 4.07 & 100 & 100 & 100 & 100 & 100 & 100 \\
\hline 2.93 & 99.9 & 99.9 & 99.9 & 100 & 99.9 & 99.9 \\
\hline 2.41 & 99.9 & 99.9 & 99.9 & 99.9 & 99.9 & 99.9 \\
\hline 2.10 & 99.9 & 99.9 & 99.8 & 99.8 & 99.9 & 99.9 \\
\hline 1.88 & 99.8 & 99.8 & 99.7 & 99.7 & 99.7 & 99.7 \\
\hline 1.72 & 99.4 & 99.4 & 99.2 & 99.3 & 99.2 & 99.3 \\
\hline 1.59 & 98.1 & 98.2 & 97.9 & 98.0 & 98.0 & 98.0 \\
\hline 1.49 & 92.5 & 93.6 & 92.9 & 91.7 & 91.9 & 92.0 \\
\hline 1.40 & 77.3 & 80.3 & 77.9 & 75.0 & 75.9 & 73.7 \\
\hline Total & 100 & 99.9 & 100 & 100 & 100 & 100 \\
\hline
\end{tabular}


Table S4 Data processing statistics (XDS version Oct 15, 2015) for different summation of frames in ins_1 data set (Figure 8).

\begin{tabular}{|c|c|c|c|c|c|c|}
\hline \multirow{2}{*}{$\begin{array}{c}\text { Resolution } \\
(\AA)\end{array}$} & \multicolumn{6}{|c|}{$R_{\text {meas }}(\%)$} \\
\hline & SUM5 & SUM10 & SUM20 & SUM40 & SUM80 & SUM160 \\
\hline 5.10 & 2.5 & 2.5 & 2.5 & 2.6 & 2.6 & 2.5 \\
\hline 3.73 & 2.4 & 2.4 & 2.5 & 2.5 & 2.5 & 2.5 \\
\hline 3.08 & 3.2 & 3.3 & 3.3 & 3.3 & 3.4 & 3.5 \\
\hline 2.68 & 5.3 & 5.4 & 5.5 & 5.4 & 5.5 & 5.7 \\
\hline 2.41 & 7.8 & 7.9 & 8.1 & 8.1 & 8.3 & 8.9 \\
\hline 2.20 & 11.9 & 12.4 & 12.6 & 12.7 & 13.3 & 14.4 \\
\hline 2.04 & 17.6 & 18.1 & 19.1 & 19.5 & 20.5 & 22.8 \\
\hline 1.91 & 29.2 & 31.7 & 34.0 & 35.9 & 38.6 & 44.3 \\
\hline 1.81 & 42.4 & 47.2 & 53.5 & 58.6 & 64.3 & 73.9 \\
\hline Total & 4.5 & 4.5 & 4.6 & 4.8 & 4.9 & 5.2 \\
\hline \multirow[t]{2}{*}{$\begin{array}{l}\text { Resolution } \\
(\AA)\end{array}$} & \multicolumn{6}{|c|}{$\langle I / \sigma(I)>$} \\
\hline & SUM5 & SUM10 & SUM20 & SUM40 & SUM80 & SUM160 \\
\hline 5.10 & 86.37 & 83.61 & 81.63 & 82.12 & 82.01 & 82.75 \\
\hline 3.73 & 87.98 & 84.95 & 82.95 & 83.25 & 83.00 & 83.57 \\
\hline 3.08 & 65.50 & 62.79 & 61.22 & 61.01 & 60.43 & 59.82 \\
\hline 2.68 & 42.70 & 41.72 & 39.65 & 39.16 & 38.40 & 37.44 \\
\hline 2.41 & 30.23 & 28.79 & 28.04 & 27.59 & 26.84 & 25.68 \\
\hline 2.20 & 19.59 & 18.51 & 17.98 & 17.59 & 16.83 & 15.80 \\
\hline 2.04 & 13.68 & 12.67 & 12.16 & 11.92 & 11.41 & 10.54 \\
\hline 1.91 & 7.83 & 7.22 & 6.71 & 6.44 & 6.08 & 5.46 \\
\hline 1.81 & 4.98 & 4.53 & 4.05 & 3.73 & 3.46 & 3.06 \\
\hline Total & 30.50 & 29.09 & 28.21 & 27.97 & 27.48 & 26.81 \\
\hline \multirow[t]{2}{*}{$\begin{array}{c}\text { Resolution } \\
(\AA)\end{array}$} & \multicolumn{6}{|c|}{$C C_{1 / 2}(\%)$} \\
\hline & SUM5 & SUM10 & SUM20 & SUM40 & SUM80 & SUM160 \\
\hline 5.10 & 99.9 & 100 & 100 & 100 & 100 & 100 \\
\hline 3.73 & 100 & 100 & 100 & 100 & 100 & 100 \\
\hline 3.08 & 99.9 & 99.9 & 99.9 & 99.9 & 100 & 100 \\
\hline 2.68 & 99.9 & 99.9 & 99.9 & 99.9 & 99.9 & 99.9 \\
\hline 2.41 & 99.8 & 99.7 & 99.7 & 99.7 & 99.7 & 99.7 \\
\hline 2.20 & 99.6 & 99.6 & 99.6 & 99.6 & 99.6 & 99.5 \\
\hline 2.04 & 99.1 & 99.1 & 99.1 & 99.1 & 99.0 & 98.8 \\
\hline 1.91 & 96.6 & 96.7 & 96.6 & 96.7 & 96.2 & 94.6 \\
\hline 1.81 & 92.6 & 91.6 & 91.8 & 91.3 & 89.3 & 86.2 \\
\hline Total & 99.9 & 100 & 100 & 100 & 100 & 100 \\
\hline
\end{tabular}


Table S5 Data processing statistics (XDS version Oct 15, 2015) for different skipping frames of lys_2 and ins_1 data sets (Figure 9).

lys_2 data

\begin{tabular}{|c|c|c|c|c|c|c|c|c|c|}
\hline \multirow[t]{2}{*}{$\begin{array}{c}\text { Resoluti } \\
\text { on ( } \AA \text { ) }\end{array}$} & \multicolumn{3}{|c|}{$R_{\text {meas }}(\%)$} & \multicolumn{3}{|c|}{$\langle I / \sigma(I)\rangle$} & \multicolumn{3}{|c|}{$C C_{1 / 2}(\%)$} \\
\hline & SUM40 & $\begin{array}{l}\text { SUM20 } \\
\text { SKIP20 }\end{array}$ & $\begin{array}{l}20 \text { SUM } \\
1 \text { SKIP1 }\end{array}$ & SUM40 & $\begin{array}{l}\text { SUM20 } \\
\text { SKIP20 }\end{array}$ & $\begin{array}{l}\text { 20SUM } \\
\text { 1SKIP1 }\end{array}$ & SUM40 & $\begin{array}{l}\text { SUM20 } \\
\text { SKIP20 }\end{array}$ & $\begin{array}{l}\text { 20SUM } \\
1 \text { SKIP1 }\end{array}$ \\
\hline 4.07 & 3 & 3.3 & 3.2 & 72.17 & 67.44 & 68.9 & 100 & 100 & 100 \\
\hline 2.93 & 3.8 & 4.1 & 4.1 & 66.71 & 61.26 & 62.08 & 100 & 99.9 & 99.9 \\
\hline 2.41 & 5.4 & 6.3 & 6.3 & 48.69 & 42.23 & 42.3 & 99.9 & 99.9 & 99.9 \\
\hline 2.10 & 7.4 & 9.0 & 9.1 & 36.03 & 30.10 & 29.89 & 99.8 & 99.8 & 99.8 \\
\hline 1.88 & 11.1 & 14.1 & 14.3 & 23.10 & 18.63 & 18.48 & 99.7 & 99.6 & 99.5 \\
\hline 1.72 & 19.3 & 24.8 & 25.0 & 12.98 & 10.51 & 10.39 & 99.3 & 98.7 & 98.6 \\
\hline 1.59 & 30.4 & 36.3 & 36.2 & 8.22 & 6.98 & 6.93 & 98.0 & 96.2 & 96.4 \\
\hline 1.49 & 45.7 & 49.4 & 49.5 & 4.38 & 3.90 & 3.83 & 91.7 & 86.5 & 87.2 \\
\hline 1.40 & 58.7 & 62.5 & 64.4 & 2.46 & 2.15 & 2.11 & 75 & 64.8 & 60.8 \\
\hline Total & 6.3 & 7.5 & 7.5 & 23.04 & 20.06 & 20.13 & 100 & 99.9 & 100 \\
\hline
\end{tabular}

ins_1 data

\begin{tabular}{|c|c|c|c|c|c|c|c|c|c|}
\hline \multirow[t]{2}{*}{$\begin{array}{l}\text { Resoluti } \\
\text { on }(\AA)\end{array}$} & \multicolumn{3}{|c|}{$R_{\text {meas }}(\%)$} & \multicolumn{3}{|c|}{$\langle I / \sigma(I)\rangle$} & \multicolumn{3}{|c|}{$C C_{1 / 2}(\%)$} \\
\hline & SUM40 & $\begin{array}{l}\text { SUM20 } \\
\text { SKIP20 }\end{array}$ & $\begin{array}{l}20 \text { SUM } \\
1 \text { SKIP1 }\end{array}$ & SUM40 & $\begin{array}{l}\text { SUM20 } \\
\text { SKIP20 }\end{array}$ & $\begin{array}{l}20 \text { SUM } \\
1 \text { SKIP1 }\end{array}$ & SUM40 & $\begin{array}{l}\text { SUM20 } \\
\text { SKIP20 }\end{array}$ & $\begin{array}{l}20 \text { SUM } \\
1 \text { SKIP1 }\end{array}$ \\
\hline 5.09 & 2.5 & 4.0 & 2.8 & 82.96 & 54.86 & 72.57 & 100 & 99.9 & 100 \\
\hline 3.72 & 2.5 & 4.4 & 2.9 & 84.05 & 55.64 & 72.26 & 100 & 99.9 & 100 \\
\hline 3.07 & 3.4 & 5.4 & 4.3 & 61.33 & 41.56 & 49.41 & 100 & 99.9 & 99.9 \\
\hline 2.68 & 5.6 & 8.2 & 7.7 & 39.41 & 27.19 & 29.85 & 99.9 & 99.8 & 99.8 \\
\hline 2.40 & 8.2 & 11.7 & 11.6 & 27.30 & 19.00 & 20.04 & 99.8 & 99.5 & 99.5 \\
\hline 2.20 & 13.1 & 18.4 & 18.6 & 17.24 & 12.00 & 12.38 & 99.6 & 99.1 & 98.9 \\
\hline 2.04 & 19.8 & 27.7 & 27.9 & 11.59 & 8.02 & 8.20 & 99.2 & 98.1 & 98 \\
\hline 1.91 & 36.4 & 49.3 & 49.8 & 6.31 & 4.36 & 4.38 & 96.7 & 92.8 & 93.7 \\
\hline 1.80 & 59.5 & 78.5 & 79.6 & 3.65 & 2.49 & 2.54 & 91 & 84.6 & 82.9 \\
\hline Total & 4.9 & 7.3 & 6.2 & 27.97 & 18.98 & 22.14 & 100 & 99.9 & 100 \\
\hline
\end{tabular}


Table S6 Data processing statistics (XDS version Oct 15, 2015) for high rotation speed data sets lys_3 - 10 (Figure 10).

\begin{tabular}{|c|c|c|c|c|c|c|c|c|}
\hline \multirow[t]{2}{*}{ Resolution $(\AA)$} & \multicolumn{8}{|c|}{$R_{\text {meas }}(\%)$} \\
\hline & 1 (SUM5) & 10 & 20 & 45 & 90 & 180 & 360 & 720 \\
\hline 4.32 & 3.2 & 3.4 & 3.5 & 3.8 & 4.0 & 4.5 & 5.8 & 7.7 \\
\hline 3.12 & 3.9 & 4.1 & 4.2 & 4.2 & 4.1 & 4.4 & 5.2 & 6.6 \\
\hline 2.57 & 5.4 & 5.5 & 5.6 & 5.7 & 5.2 & 5.3 & 5.5 & 6.1 \\
\hline 2.23 & 7.5 & 7.4 & 7.7 & 7.7 & 7.2 & 7.1 & 7.2 & 7.9 \\
\hline 2.00 & 10.2 & 10.0 & 10.7 & 10.6 & 9.9 & 9.8 & 9.9 & 11.0 \\
\hline 1.83 & 16.5 & 15.7 & 17.0 & 18.2 & 17.1 & 17.0 & 17.0 & 19.1 \\
\hline 1.69 & 26.9 & 25.0 & 26.7 & 31.7 & 30.7 & 31.8 & 31.0 & 35.2 \\
\hline 1.59 & 39.4 & 37.6 & 38.9 & 49.3 & 52.0 & 54.4 & 53.1 & 60.5 \\
\hline 1.50 & 50.4 & 52.8 & 53.8 & 71.1 & 86.2 & 90.3 & 93.0 & 108.1 \\
\hline Total & 6.9 & 6.8 & 7.2 & 7.5 & 7.4 & 7.7 & 8.6 & 10.2 \\
\hline \multirow[t]{2}{*}{ Resolution $(\AA)$} & \multicolumn{8}{|c|}{$\langle I / \sigma(I)\rangle$} \\
\hline & 1 (SUM5) & 10 & 20 & 45 & 90 & 180 & 360 & 720 \\
\hline 4.32 & 65.8 & 62.82 & 60.87 & 58.34 & 59.72 & 54.30 & 45.76 & 36.99 \\
\hline 3.12 & 64.85 & 62.35 & 60.09 & 57.96 & 59.8 & 55.24 & 47.55 & 38.88 \\
\hline 2.57 & 48.73 & 47.61 & 45.27 & 43.78 & 45.6 & 43.54 & 40.06 & 33.94 \\
\hline 2.23 & 36.44 & 36.99 & 34.83 & 33.54 & 35.13 & 34.31 & 32.69 & 28.9 \\
\hline 2.00 & 26.43 & 27.34 & 25.4 & 24.18 & 25.56 & 25.36 & 25.08 & 22.84 \\
\hline 1.83 & 15.69 & 16.66 & 15.74 & 14.45 & 15.3 & 15.36 & 15.8 & 14.77 \\
\hline 1.69 & 9.48 & 9.93 & 9.65 & 8.41 & 8.75 & 8.78 & 9.26 & 8.81 \\
\hline 1.59 & 6.29 & 6.48 & 6.38 & 5.37 & 5.24 & 5.20 & 5.66 & 5.38 \\
\hline 1.50 & 3.51 & 3.39 & 3.42 & 2.8 & 2.48 & 2.44 & 2.60 & 2.45 \\
\hline Total & 23.80 & 23.81 & 22.72 & 21.45 & 22.20 & 21.30 & 20.06 & 17.56 \\
\hline \multirow[t]{2}{*}{ Resolution $(\AA)$} & \multicolumn{8}{|c|}{$C C_{1 / 2}(\%)$} \\
\hline & 1 (SUM5) & 10 & 20 & 45 & 90 & 180 & 360 & 720 \\
\hline 4.32 & 100 & 100 & 99.9 & 99.9 & 99.9 & 99.8 & 99.7 & 99.4 \\
\hline 3.12 & 99.9 & 99.9 & 99.9 & 99.9 & 99.9 & 99.8 & 99.8 & 99.6 \\
\hline 2.57 & 99.9 & 99.9 & 99.9 & 99.9 & 99.9 & 99.9 & 99.9 & 99.8 \\
\hline 2.23 & 99.8 & 99.8 & 99.8 & 99.8 & 99.8 & 99.8 & 99.8 & 99.8 \\
\hline 2.00 & 99.7 & 99.8 & 99.7 & 99.7 & 99.7 & 99.8 & 99.7 & 99.7 \\
\hline 1.83 & 99.4 & 99.4 & 99.4 & 99.3 & 99.5 & 99.5 & 99.5 & 99.4 \\
\hline 1.69 & 98.1 & 98.6 & 98.4 & 98.2 & 98.4 & 98.4 & 98.5 & 98.1 \\
\hline 1.59 & 93.1 & 95.0 & 94.4 & 94.2 & 94.6 & 94.2 & 94.6 & 93.8 \\
\hline 1.50 & 77.4 & 80.0 & 78.4 & 75.3 & 75.9 & 77.4 & 76.3 & 73.8 \\
\hline Total & 100 & 99.9 & 99.9 & 99.9 & 99.9 & 99.9 & 99.9 & 99.8 \\
\hline
\end{tabular}

lys_3 - 10 data sets processed with same $0.9^{\circ}$ rotation angle

\begin{tabular}{|c|c|c|c|c|c|c|c|c|}
\hline \multirow{2}{*}{$\begin{array}{c}\text { Resolution } \\
(\AA)\end{array}$} & \multicolumn{8}{|c|}{$R_{\text {meas }}(\%)$} \\
\hline & 1 & 10 & 20 & 45 & 90 & 180 & 360 & 720 \\
\hline 4.32 & 3.1 & 3.1 & 3.2 & 3.5 & 4.0 & 4.6 & 5.9 & 7.7 \\
\hline 3.12 & 3.8 & 3.8 & 3.8 & 3.9 & 4.2 & 4.6 & 5.4 & 6.6 \\
\hline 2.57 & 5.7 & 5.4 & 5.5 & 5.5 & 5.4 & 5.6 & 5.7 & 6.1 \\
\hline 2.23 & 8.3 & 7.8 & 7.9 & 7.8 & 7.8 & 7.8 & 7.8 & 7.9 \\
\hline 2.00 & 12.6 & 11.4 & 11.8 & 11.3 & 11.3 & 11.2 & 11 & 11 \\
\hline 1.83 & 23.3 & 20.9 & 21.4 & 20.7 & 20.7 & 20.7 & 19.5 & 19.1 \\
\hline 1.69 & 44.5 & 38.6 & 40.2 & 38.5 & 38.3 & 39.4 & 36.1 & 35.2 \\
\hline 1.59 & 75.5 & 66.7 & 68.8 & 65.3 & 66.2 & 67.3 & 61.7 & 60.5 \\
\hline 1.50 & 127.9 & 114 & 118.5 & 113.5 & 114.1 & 117.2 & 109.2 & 108.1 \\
\hline
\end{tabular}




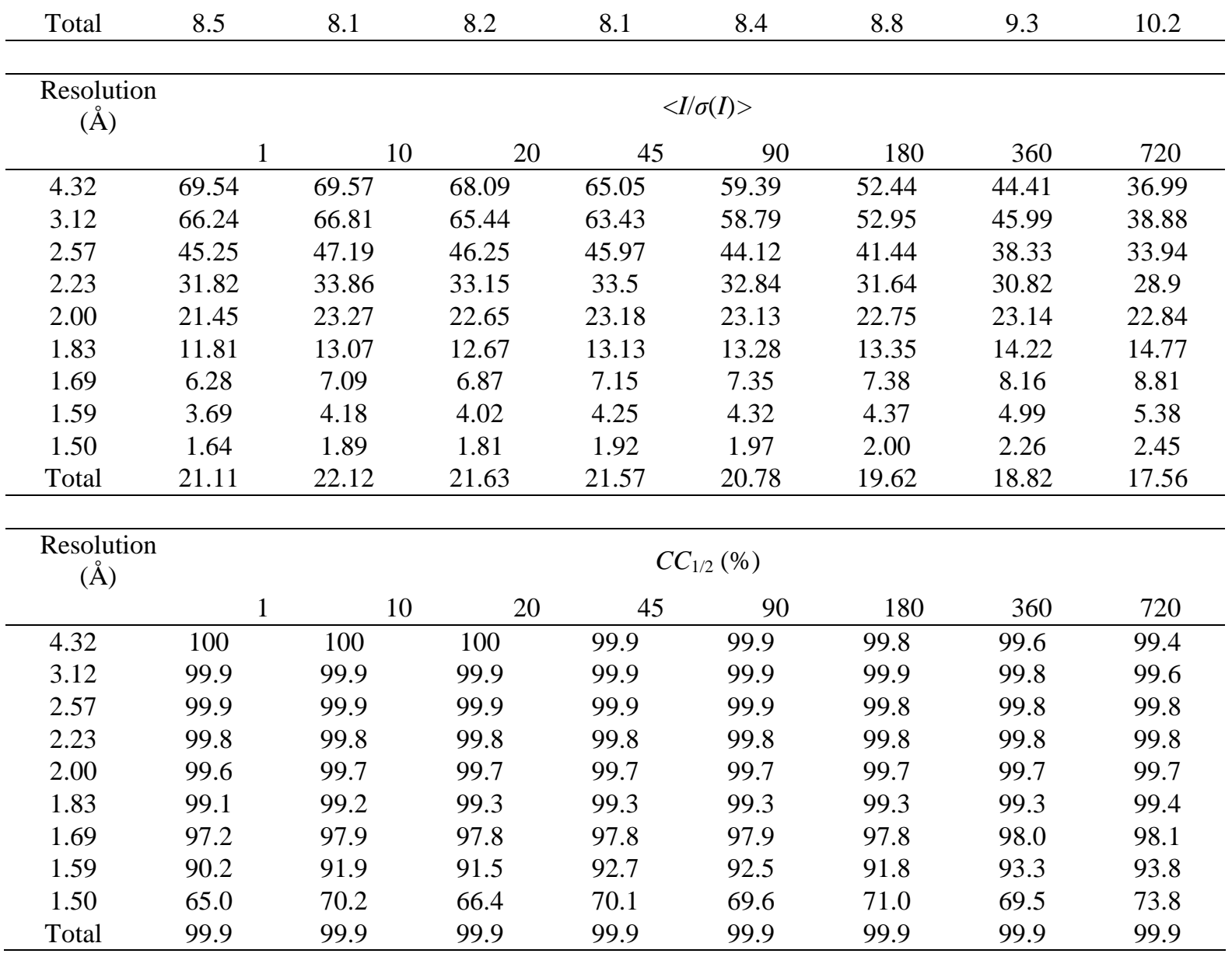


Table S7 Data processing statistics (XDS version Oct 15, 2015) for one second native-SAD data set ins_2 (Suppl. Figure 1).

\begin{tabular}{cccc}
\hline Resolution $(\AA)$ & $R_{\text {meas }}(\%)$ & $\langle I / \sigma(I)\rangle$ & $C C_{1 / 2}(\%)$ \\
\hline 6.81 & 2.2 & 62.86 & 99.9 \\
4.85 & 2.1 & 59.52 & 99.9 \\
3.97 & 2.2 & 55.75 & 99.9 \\
3.45 & 2.6 & 47.17 & 99.9 \\
3.08 & 3.9 & 32.67 & 99.8 \\
2.82 & 5.9 & 19.53 & 99.6 \\
2.61 & 9.4 & 10.91 & 99.8 \\
2.44 & 13.4 & 6.05 & 97.3 \\
2.30 & 24.4 & 2.99 & 91.9 \\
total & 2.8 & 29.59 & 99.9 \\
\hline
\end{tabular}


Figure S1 One second native-SAD experiment. A quadrant of an EIGER 16M was used to simulate a EIGER 4M(16M) detector. A one second data collection time native-SAD experiment was attempted with an insulin crystal (data set insu_2). The $C C_{\text {all }}$ versus $C C_{\text {weak }}$ in SHELXD and contrast versus cycle of density modification in SHLXE are depicted.

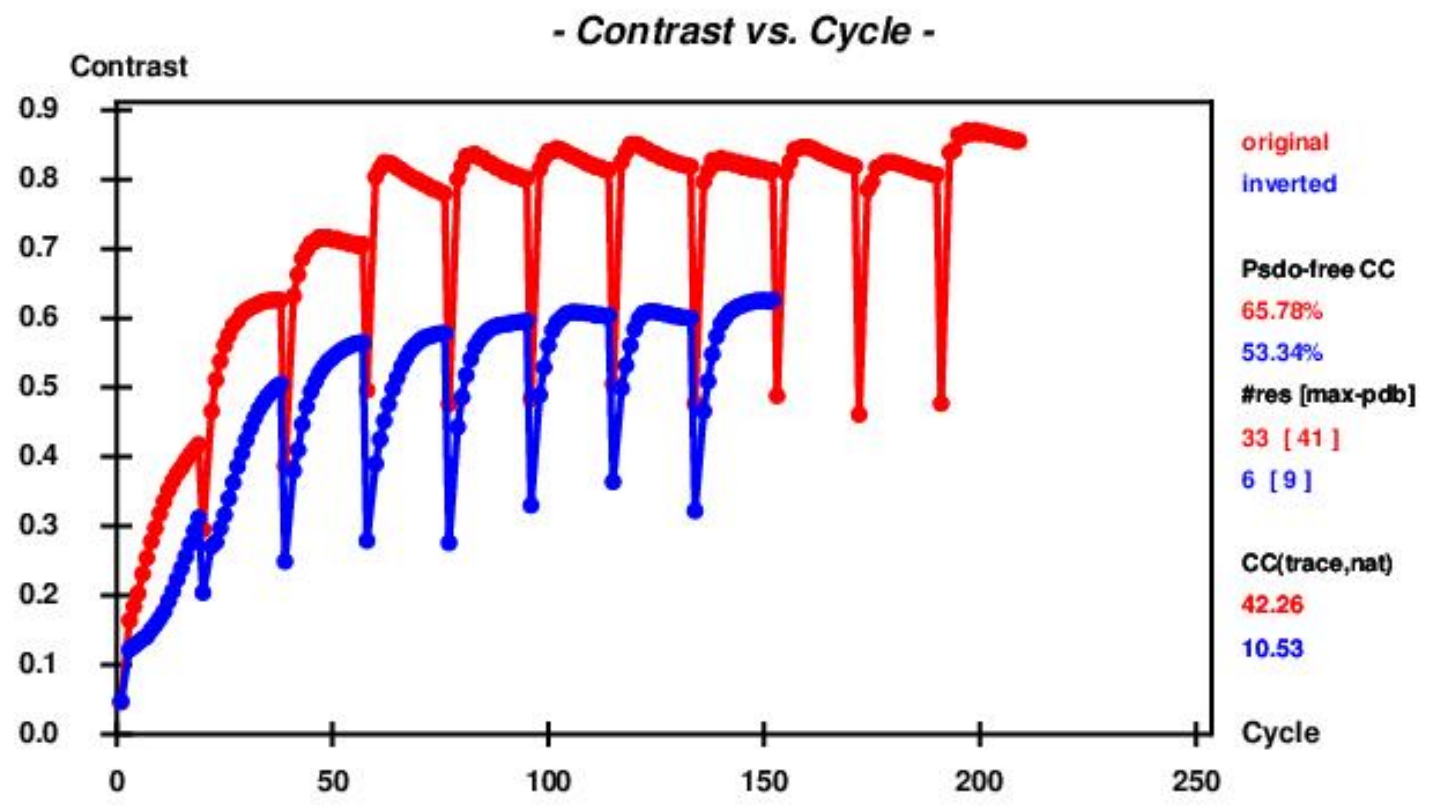

(a)

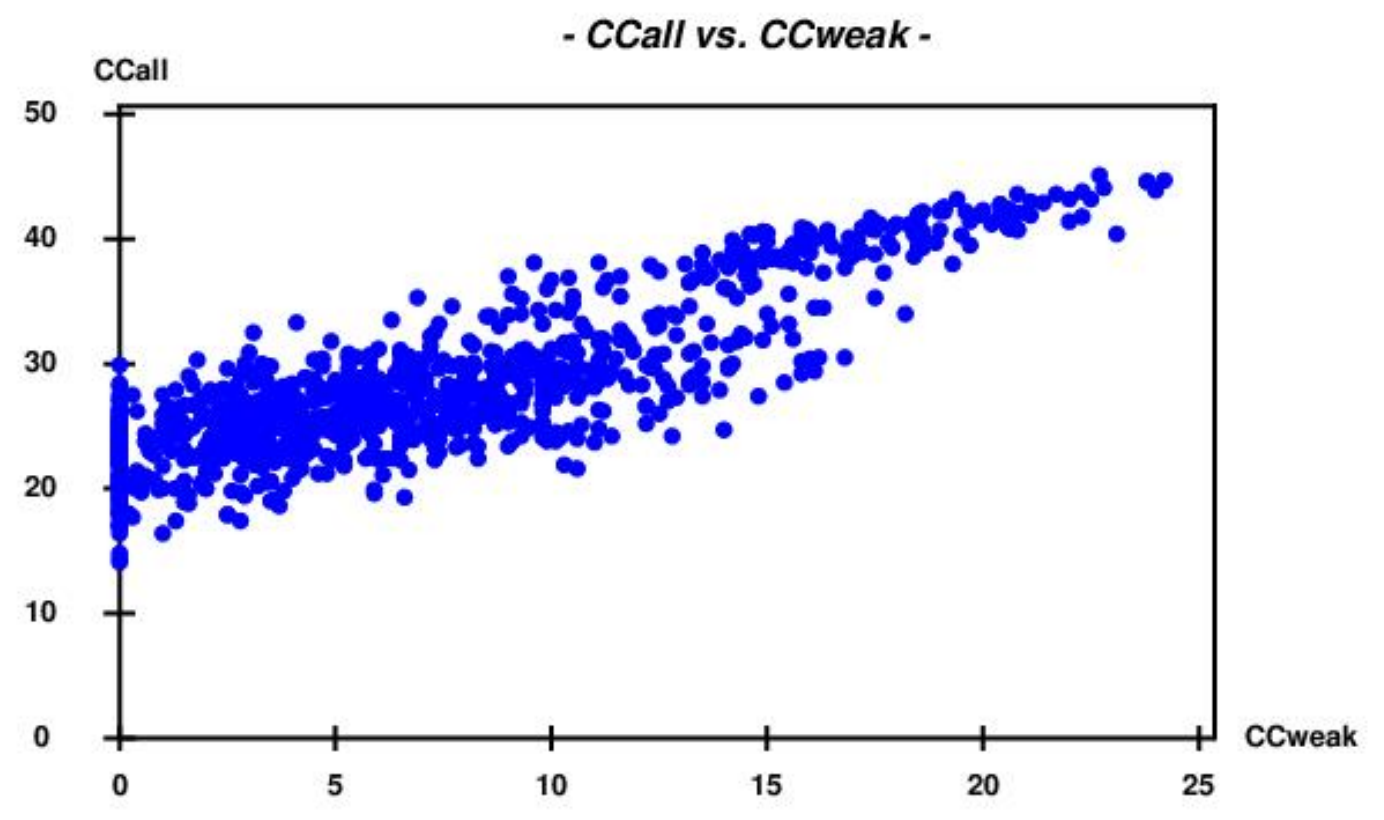

(b) 